\title{
All zones arthroscopic meniscal repair - medium term follow-up
}

\author{
Chitta Ranjan Sahu ${ }^{1, *}$, Deepak $K^{2}$, Pavan Kumar Yadav Kondepu ${ }^{3}$, Gudaru Jagadesh ${ }^{4}$ \\ ${ }^{\mathbf{1 , 2}}$ Assistant Professor, ${ }^{3}$ Resident, ${ }^{\mathbf{4}}$ Director and HOD, Dept. of Orthopaedics, Balaji Institute of Surgery Research and \\ Rehabilitation for the Disabled (BIRRD) (T), TTD, Tirupati, Andhra Pradesh, India
}

\section{*Corresponding Author:}

Email: chittusahu@gmail.com

\begin{abstract}
Introduction: The menisci are crucial structures of the knee in maintaining function. Meniscal repair has gained importance as meniscectomy causes early arthritis. Arthroscopic repair of meniscus has become the mainstay of management replacing open surgery, because of decreased duration of surgical procedure and better accessibility to the tear location.

Methods: This study prospectively evaluated functional outcome in 34 patients who underwent arthroscopic meniscal repair. Tears involving outer (red-red), middle and inner (white-white) zone were included for repair. Complex and degenerative tears were excluded from repair. Follow-up consisted of clinical assessment by using Barrett's criteria and comparison of pre-operative and post-operative Lysholm knee score. In the absence of joint-line tenderness or effusion, and if the McMurray test was negative, it was considered that the repaired meniscus has healed.

Results: After a minimum of 1.5 year follow-up, 32/34 of the cases that underwent meniscal repair were asymptomatic for meniscal tears. The mean Lysholm score increased to 95.2 as compared with the preoperative mean value of 61 . Arthroscopic meniscal repair was not associated with any neurovascular or other major complications.

Conclusion: Stabilizing the meniscal tear by arthroscopy in all three zones appears to be an effective procedure.
\end{abstract}

Keywords: Meniscal repair, White-white zone, Arthroscopy.

\section{Introduction}

The menisci are crucial structures of the knee and contribute to the maintenance of normal knee function and joint stability. The outer $20 \%$ to $30 \%$ of the medial meniscus and $10 \%$ to $25 \%$ of the lateral meniscus are vascular. ${ }^{1}$ The outer red-red zone, is well vascularized and has a good healing capacity, while the intermediate red-white zone and the innermost white-white zone have poor intrinsic healing owing to their avascular nature. ${ }^{1}$ In the early years of meniscal surgery, complete excision of the injured meniscus was the gold standard of treatment. It was soon recognised that a total or subtotal meniscectomy leads to development of osteoarthritis inevitably within 5-10 years after surgery. ${ }^{2,3}$ In 1889 , Annandale ${ }^{4}$ reported on the first successful meniscal repair. However, meniscal repair became popular only in the late 1970s. De Haven ${ }^{5}$ popularized open meniscal repair as an alternative to meniscectomy.

The heterogeneity of meniscal tears restricts the indications for meniscal repair. The site of the lesion is an important aspect, lesion must be in more vascularized peripheral region of the meniscus (red zone) in order to facilitate the healing process. Over a period of several months, the healed tissue matures to a fibrocartilage. ${ }^{6}$ There is a vast array of surgical options to choose from. Numerous techniques have been described. Newer devices and implants are constantly evolving. The three most commonly used methods of arthroscopic meniscal repair are-; 1. Inside-out; 2. Outside-in and ; 3. All inside technique. Both inside-out and outside-in repair techniques involve passing a suture from either the inside or the outside of the knee via arthroscopy and tied beyond the joint capsule using a mini incision. These techniques are particularly useful for anterior and middle third tears which are not easily accessed by an all-inside technique, which is reserved for middle and posterior tears. Arthroscopic guided meniscal repair has multiple advantages over open repair, such as smaller surgical incision, less postoperative pain, fewer adhesion and earlier mobilization.

The purpose of this study was to evaluate the medium-term clinical outcome in patients who had undergone isolated arthroscopic repair of medial or lateral meniscal tears, or meniscal repair in combination with anterior cruciate ligament (ACL) reconstruction in a consecutive series of 34 patients. The study hypothesis was that arthroscopic meniscal repair and stabilizing the tear in all three zones appears to be an effective procedure.

\section{Method}

From 15 March 2016 to 15 November 2016, all the patients who underwent surgical management of meniscus during the duration of study.

\section{Inclusion Criteria:}

1. Symptomatic tears

2. Peripheral, red-red, red-white and white-white tears

3. Non deformed and repairable

4. Unstable: $>3 \mathrm{~mm}$ of excursion

\section{Exclusion Criteria:}

1. Degenerative tears and clinically non-correlative tears in older patients

2. Stable tears $<5 \mathrm{~mm}$ in length

3. Stable, incomplete tears

4. Associated infections, rheumatoid or other collagen vascular diseases and late osteoarthritis. 
Postoperative Evaluation: All the patients were evaluated periodically at 3weeks, 6weeks, 3months, 6 months and then yearly. The minimum period of follow up was 1.5 years. At each follow-up clinical evaluation was done with Lysholm score questionnaire and Barrett's criteria.

Surgical Technique: All cases were done under spinal anaesthesia in the supine position with foot rest at 90 degrees of knee flexion and thigh support. Pneumatic tourniquet was applied to the proximal thigh after proper padding with pressure set to $200 \mathrm{~mm}$ of $\mathrm{Hg}$ more than the systolic blood pressure. A diagnostic round of arthroscopy was performed initially. Knee was entered through the anterolateral viewing portal which was made slightly higher than the conventional portal to escape the fat pad. The anteromedial portal was made appropriately under arthroscopic guidance with the help of a needle. The morphology of the meniscus tear, measurement of the tear length and the rim width, and other ligamentous injuries were confirmed. Whenever a dislocated or a locked bucket-handle tear was encountered, reduction of the tear was first performed. To stimulate the healing process bleeding was encouraged at the periphery of the meniscus by making multiple perforations by the help of micro-fracture awls. For stabilization of the torn meniscus by all inside technique, Fast-fix (Smith and Nephew) suture anchors were used. Each Fast-Fix device is made up of two 5$\mathrm{mm}$ polymer suture-bar anchors with a pre-tied selfsliding knot of No. 0 non-absorbable USP braided polyester suture material. In addition, a split cannula facilitates easy insertion of the device into the knee joint, and functions as a depth penetration limiter. The torn edges of the meniscus to be stabilized were first freshened. Following, the distance of the tear from the periphery was measured by a depth gauge. The depth limiter in the Fast-fix device was then trimmed accordingly to avoid inadvertent advancement of the Fast-fix and neurovascular injury. The first bite was always taken close to the periphery and the second bite at the inner segment of the meniscus by gently withdrawing the needle which caused the polymer suture bars to flip automatically. The bites were taken in the said manner because the meniscus is stabilized from the second to the first bite. After the second bite the delivery needle was removed from the knee joint keeping the end of the suture free. A knot pusher was then inserted on the free end of the suture to slide the self-sliding knot by creating tension on the suture. Following, the suture was cut by a cord cutter (Fig. 1). In case the patient was diagnosed to also have a concomitant ACL injury, arthroscopic ACL reconstruction was performed after the meniscal repair was completed. All the ACLs were reconstructed by hamstring graft, using suspensory fixation on the femoral side and bio-interference screw on the tibial side. Portals and graft donor site was then sutured in layers.

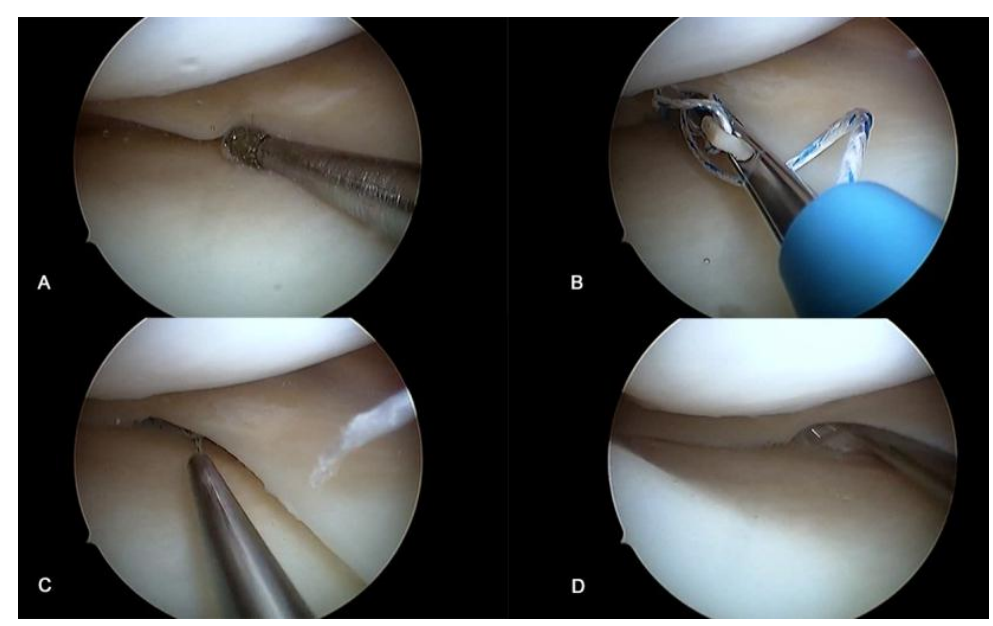

Fig. 1: Steps of all inside meniscal repair. A. Debridement of the torn edges; B. Deployment of all inside meniscal anchor; C. Tightening of the knot by a knot pusher cum cutter; D. Checking stability of the repaired meniscus

Postoperative Rehabilitation: After surgery, all the patients who underwent meniscal repair were kept nonweight-bearing on the affected limb for 6 weeks. Full weight bearing was permitted at 6 weeks postoperatively. Knee brace was used for all patients for the first 2 weeks. Non- weight-bearing range of movement exercises were started in immediate post-operative period. Knee flexion range was gradually allowed to 90 degrees with help motion control brace. After 6weeks, range of motion exercises were started to get back the complete range of motion. Jogging and climbing stairs was permitted after 12 weeks. Unrestricted activity was permitted at 6 months for patients with isolated meniscal repair. Patients who underwent meniscal repair and an ACLreconstruction also followed the same rehabilitation protocol. Knee brace was continued till 8 weeks. Unrestricted activity \& contact sports were permitted only after1 year. 
Analysis of Results: SPSS software version 14 was used to do the statistical analysis. The preoperative and postoperative Lysholm scores were compared by paired-t test.

\section{Results}

There were 34 (32 male, 2 female) patients in this prospective series with 36 injured menisci. None of the patients in this series were lost to follow-up. The average age at the time of meniscal repair was 29 years (range, 15-44years). All the patients were followed up for an average 20.23 months (15-23months). In 22 $(65 \%)$ meniscal tears, injury-to-repair interval was $\leq 3$ weeks. These were grouped as acute tears. In 12(35\%) tears, injury-to repair interval was $>3$ weeks. These were grouped as chronic tears. Isolated meniscal tears which were repaired were seen in $9(26 \%)$ and meniscal tears which were repaired in combination with ACL reconstruction was seen in $25(74 \%)$ knees. There were $12(33.33 \%)$ tears in the red-red zone, $21(58.33 \%)$ tears in the red-white zone and $3(8.33 \%)$ tears in the whitewhite zone (Fig. 2).

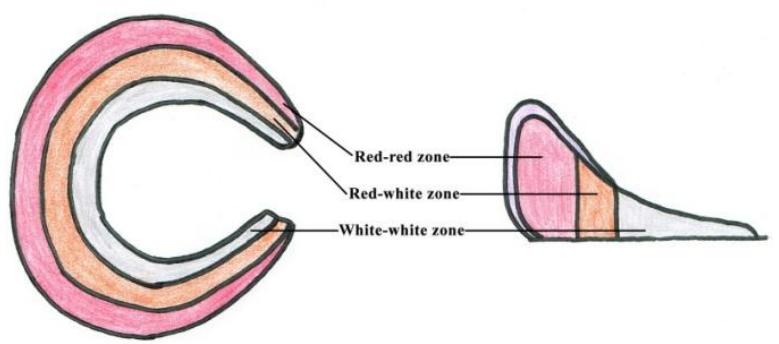

Fig. 2: Diagrammatic representation of different zones of the meniscus

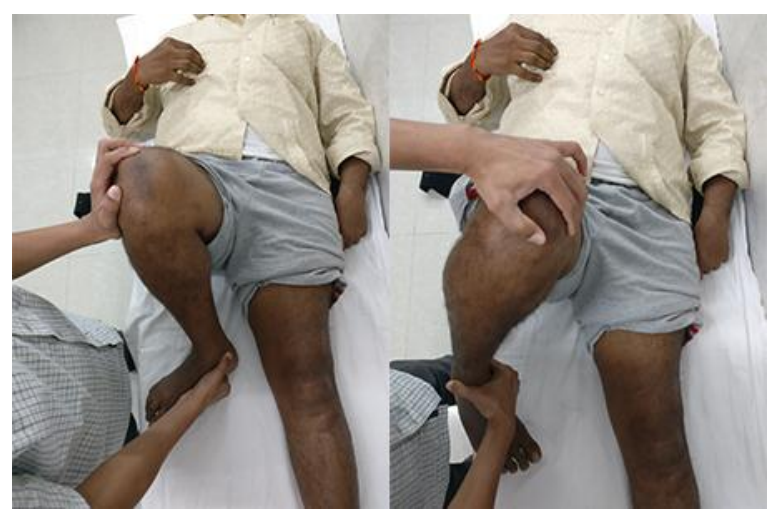

Fig. 3: Clinical demonstration of Mc murrays test

No symptoms of meniscal tears were observed in 32 of the 34 cases (Table 1) in their last follow-up which also included 3 cases of meniscal repair done in the white-white zone. Hence, $94 \%$ cases were considered as clinically healed. Of the other two cases, one patient had joint line tenderness, and the other had a positive McMurray's test (Fig. 3) during follow-up. None of the patients had any locking episodes. The overall Lysholm score increased to a mean value of 95.2 as compared with the preoperative mean value of 61.5 ( $\mathrm{p}<0.0001$ ). In 9 cases where isolated meniscal repair was done, the Lysholm score on an average increased to $97 \%$ postoperatively from a preoperative score of $65.8 \%$ (Table 2). In the 25 cases where ACL reconstruction was combined with meniscal repair, the Lysholm score increased from $60.0 \%$ preoperative to 94.5\% postoperative (Table 3 ).

Table 1: Barrett's criteria showing the post-operative healing

\begin{tabular}{|c|c|c|}
\hline Barrett's Criteria & Pre-operative $(n=34)$ & Post-operative positive criteria $(n=34)$ \\
\hline Joint line tenderness & 26 & 1 \\
\hline Effusion & 7 & 0 \\
\hline McMurray test & 32 & 1 \\
\hline
\end{tabular}

Table 2: Results of isolated meniscal repair

\begin{tabular}{|l|c|c|}
\hline Isolated Meniscal repair & $\begin{array}{c}\text { Preoperative Lysholm } \\
\text { score }\end{array}$ & Post-operative Lysholm score \\
\hline Chih-Wei Chiang, et al & 65 & 95 \\
\hline Present study & 65.8 & 97 \\
\hline
\end{tabular}

Table 3: Results of meniscal repair with concurrent ACL reconstruction

\begin{tabular}{|l|c|c|}
\hline ACLR+ Meniscal repair & Preoperative Lysholm score & Post-operative Lysholm score \\
\hline Chih-Wei Chiang, et al & 62 & 88 \\
\hline Present study & 60 & 94.5 \\
\hline
\end{tabular}

Table 4: Comparison of clinical success of meniscal repair with other studies

\begin{tabular}{|l|c|c|c|c|c|}
\hline Study & $\begin{array}{c}\text { Sample } \\
\text { size }\end{array}$ & $\begin{array}{c}\text { Follow-up } \\
\text { duration }\end{array}$ & $\begin{array}{c}\text { Pre-operative } \\
\text { score }\end{array}$ & $\begin{array}{c}\text { Post-operative } \\
\text { score }\end{array}$ & Clinical success \\
\hline Kotsovolos et al. & 61 & 18 months & - & - & $90 \%(55 \mathrm{healed})$ \\
\hline S. Perumal et al & 24 & 30 months & - & - & $91 \%(22 \mathrm{healed})$ \\
\hline
\end{tabular}




\begin{tabular}{|l|c|c|c|c|c|}
\hline Chih-Wei Chiang, et al & 31 & 36 months & 65.7 & 90.4 & $96.8 \%$ \\
\hline Barrett et al & 51 & 24 months & - & - & $88 \%$ \\
\hline Andrew et al. & 37 & 24 months & - & - & $86.4 \%$ (32 healed) \\
\hline Present study & 34 & 6 months & 61.5 & 95.2 & $94 \%$ (32 healed) \\
\hline
\end{tabular}

\section{Discussion}

Meniscal healing is mainly dependent on the blood supply to the meniscus. Inner $1 / 3$ of each meniscus lies in the avascular zone and is nourished by diffusion of synovial fluid. The middle $1 / 3$ obtains dual nourishment from both the blood and synovial fluid. A healing response similar to that of other connective tissues is produced in the peripheral third of the meniscus due to its rich vascular supply, has been supported by many recent studies. This tissue over a period of several months, gradually matures to fibrocartilage following the completion of the healing process. ${ }^{6,7}$

Arthroscopic meniscal repair has the advantages of decreased surgical time, decreased tissue damage and is easy to perform when compared to the open technique. Hence, this technique has become the procedure of choice in the treatment of meniscus repair. There are numerous varieties of meniscal repair devices available now including meniscal arrows, darts, screws, staples and other suture devices.

As the white-white zone does not receive a direct blood supply, it was thought that meniscal repair in this area would not be successful. But, to the contrary, repair in this area showed clinical success in our study. Repair of the meniscus in the white-white zone in 3 patients, had excellent outcome in the medium term follow-up.

In an evidence-based review performed by Jesus et al., about the outcomes of all-inside meniscal repair devices, the failure rates were found to range from $0 \%$ to $43.5 \% .{ }^{8}$ Meniscus arrow had the success rate ranging from $88 \%$ to $95 \%$, according to the most recent studies. Asik $\mathrm{M}$ et al. reported the healing rate to be close to 90\% with the T-Fix system, whereas in our study the success rate was $94.11 \%$.

S. Perumal et al reviewed their meniscal repair cases with an average of 2 years follow-up. In the 22 of 24 cases in their study, no symptoms of meniscal tears were found. ${ }^{9}$ In our study 32 of 34 cases were found asymptomatic at an average of 20.23 months follow-up.

Kotsovolos et al. used the Fast-Fix meniscal repair system and reported the clinical results of 61 menisci repaired after an average follow-up period of 18 months. ${ }^{10}$ In their series the success rate was $90 \%$ (55 clinically healed menisci out of 61) according to the criteria of Barrett et al. Fifty-one patients (88\%) had an excellent or good result. ${ }^{11}$ In the study done by Andrew et al., Fast-Fix was used for 47 meniscal tears in 37 patients. At a minimum follow-up of at least a 2-years, five of their cases were considered as clinical failure ${ }^{12}$ (Table 4).

Previous meniscal repair studies revealed success rates of $91 \%$ and $80 \%$ in patients with and without concurrent ACL reconstruction, respectively. ${ }^{14}$ But in our present study, success rates of isolated meniscal repair is $100 \%$ and success in meniscal repair with concurrent ACL reconstruction is $92 \%$, this may be because of short follow-up and a less number of cases.

Isolated meniscal repair in 9 cases with Lysholm score averaging 65.8 preoperatively showed an increased score postoperatively to $97 \%$. Meniscal repair along with ACL reconstruction done in 25 cases, showed an increase in Lysholm score from $60.0 \%$ preoperatively to $94.5 \%$ postoperatively.

Past studies have showed that there is an increased risk of arthrofibrosis in meniscal repair with concurrent ACL reconstruction. ${ }^{15}$ Twenty five patients in the present study underwent meniscal repair with concurrent ACL reconstruction. There were no patients who complained of any episode of giving way or difficulty in movement. These results demonstrate that ACL reconstruction at the time of meniscal repair achieves stability and successful knee function.

The main drawbacks of the present study are the small number of cases, the lack of a control group and the limited follow-up. There were no complications directly associated with the implant in the present series, such as synovitis, breakage or migration of the implants. The most important concern associated with the learning curve of this technique was obtaining a perfect angle to reach the desired points on the meniscus and avoiding penetration of the superficial structures including the skin. Measurement of the desired depth using a meniscal depth probe followed by trimming of the depth-limitation device should be done to avoid over penetration and thereby causing iatrogenic injuries. Therefore, understanding and correct usage of the instrument and device is important for every surgeon.

Post-operative MRI of knee joint was done after 6 weeks in ten patients who underwent arthroscopic meniscal repair. This showed that the repair was stable with no new tear patterns. Second look arthroscopy was performed in two cases (patients were asymptomatic and operated for other reason) which showed the repaired meniscus to be stable in both the cases.

\section{Conclusion}

Current study reiterates the role of arthroscopic meniscal repair as an effective procedure with high success rate. Functional outcome following meniscal repair shows significant improvement as measured by Lysholm knee score \& Barrett's criteria for meniscal healing. This is also true for the meniscal repair done in the white-white zone and concurrent meniscal repair with ACL reconstruction. 


\section{References}

1. Arnoczky SP, Warren RF. Microvasculature of the human meniscus. Am J Sports Med. 1982;10:905.

2. Fairbank TJ. Knee joint changes after meniscectomy. $J$ Bone Joint Surg Br. 1948;30B:66470.

3. Jackson JP. Degenerative changes in the knee after meniscectomy. BrMed J. 1968;2:5257.

4. Annandale T. Excision of the internal semilunar cartilage, resulting in perfect restoration of the joint movements. $\mathrm{Br}$ Med J. 1889;1:291-292.

5. DeHaven KE. Peripheral meniscus repair: An alternative to meniscectomy. Orthop Trans. 1981;5:399-400.

6. Maitra RS, Miller MD, Johnson DL. Meniscal reconstruction: Part I: Indications, techniques, and graft considerations. Am J Orthop. 1999;28:213-8.

7. Newman AP, Daniels AU, Burks RT. Principles and decision making in meniscal surgery. Arthrosc. 1993;9:33-51.

8. Jesus L, Benjamin M, Dilworth C. All inside meniscus repair: A systemic review. Clin Orthop Relat Res. 2006;455:134-41.

9. Perumal $\mathrm{S}$ et al. Clinical results of arthroscopic all-inside meniscal repair. Int J Res Med Sci. 2016 Nov;4(11):49654969.

10. Kotsovolos ES, Hantes ME, Mastrokalos DS. Results of all-inside meniscal repair with the FasTFix meniscal repair system. Arthrosc. 2006;22:3-9.

11. Barrett GR, Field MH, Treacy SH, Ruff CG. Clinical results of meniscus repair in patients 40 years and older. Arthrosc. 1998;14:824-9.

12. Andrew L, Anthony A, Joshua H. Meniscal repair using FasT-Fix all-inside menical de vices. Arthrosc. 2005;21:167-75.

13. Chih-Wei Chiang, et al., Arthroscopic all-inside meniscal repair. Chang Gung Med J Vol. 34 No. 3 May-June 2011.

14. Haas AL, Schepsis AA, Hornstein J, Edgar CM. Meniscal repair using the FasT-Fix all-inside meniscal repair device. Arthroscopy. 2005;21:167-75.

15. Austin KS, Sherman OH. Complications of arthroscopic meniscal repair. Am J Sports Med 1990;21:864-9. 